\title{
Detection of Calcification form Mammogram Image using Canny Edge Detector
}

\author{
M. Manikandan, M. Paranthaman and B. Neeththi Aadithiya \\ Department of Electronics and Communication Engineering, M. Kumarasamy College of Engineering \\ (Autonomous), Karur - 639113, Tamil Nadu, India; maniece022@gamil.com, \\ paranthaman765@gmail.com, neethiece@gmail.com
}

\begin{abstract}
Objectives: To help the physician to identify the amount of calcium in the breast cells as part of cancer detection. Methods/ Statistical Analysis: In this paper we compare the analysis of two different algorithms: Watershed method of identifying the cancer cells and edge detection by canny edge detection. The comparison was taken for the same mammogram image sequence. Initially, preprocessing like filtering and resizing was done for the mammogram images. Preprocessed images were appended to the cancer affected location by edge detection by canny edge detector. It used for identifying the depth of the calcification in a mammogram image sequence. This is turn into the appropriate examination. Findings: The watershed algorithm produces the entire calcium affected areas with major super imposed color. Whereas, in canny edge detector the minor cells were highly identified by the calcium affected cells. This would help physician for appropriate treatments. Application/ Improvements: This is the best algorithm for the distribution of calcification over a distance.
\end{abstract}

Keywords: Cancer Depth, Canny Edge, Mammogram, Micro-calcification, Sobel Mask

\section{Introduction}

Breast cancer most commonly develops in cells from the lining of milk ducts and the lobules that supply the ducts with milk. The balance of benefits versus harms of breast cancer screening is controversial. Breast cancer screening refers to testing otherwise-healthy women for breast cancer in an attempt to achieve an earlier diagnosis under the assumption that early detection will improve outcomes. A number of screening tests have been employed including clinical and self breast exams, mammography, genetic screening, ultrasound, and magnetic resonance imaging. In a number of breast pathologies, calcium is often deposited at sites of cell death or in association secretions or hyalinized stroma, resulting in pathologic calcification. For example, small, irregular, linear calcifications may be seen, via mammography, in a ductal carcinoma-in-situ to produce visible radio-opacities.

From a chemical point of view, it is interesting to notice that two types of breast calcifications have been described one is Micro calcification and the other one is Macro calcification. The micro calcification where calcium is in the lowest form. It will be distributed randomly. In macro calcification the lager amount of calcium will be covered in the breast tissues and identification of these types of cancers is quite tough for the physician. Here we answer for those problem by differentiating the kind of calcification so that it will be easier for the physician for further examination. The manual identification of the mammogram images leads to wrong diagnosis. To avoid such issues, the automatic identification of calcification can be done using CAD tools. It will play a vital role in the field of Medical imaging.

\section{Existing Work in Breast Cancer from Mammogram}

The micro calcification detection in breast cancer can be carried over by many techniques. We used the novel 
approach involving watershed method for the identification of Calcifications in breast cancer image ${ }^{11-15}$. In picture preparation, a watershed is a change portrayed on a grayscale picture. The name alludes allegorically to a land watershed, or seepage isolate, which isolates abutting waste bowls ${ }^{1}$. The watershed change treats the photograph it works upon like a topographic guide, with the splendor of each point speaking to its top, and uncovers the lines that keep running close by the highest points of edges $^{8-10}$. There are particular specialized meanings of a watershed. In diagrams, watershed follows can be characterized at the hubs, on the edges, or half breed follows on every hub and edges. Watersheds may likewise be characterized inside the relentless space. There likewise are numerous particular calculations to process watersheds ${ }^{1}$.

Watershed algorithm is used in photograph processing broadly speaking for segmentation function. Watershed set of guidelines is considered as a capable apparatus for picture division. It plays a pivotal position in device inventive and insightful, video picture division and photograph examination. The straight forward thought of the watershed set of guidelines is to see the inclination of a grayscale picture as a topographic floor, in which the rain falling at the watershed line could be gathered similarly in catchments bowls. Vincent and Soiled proposed the radical approach for finding the watershed strains by utilizing the utilization of the submersion reproduction calculation. Division viability of the watershed revise is progressed broadly if frontal area items and history territories are stamped as of now.

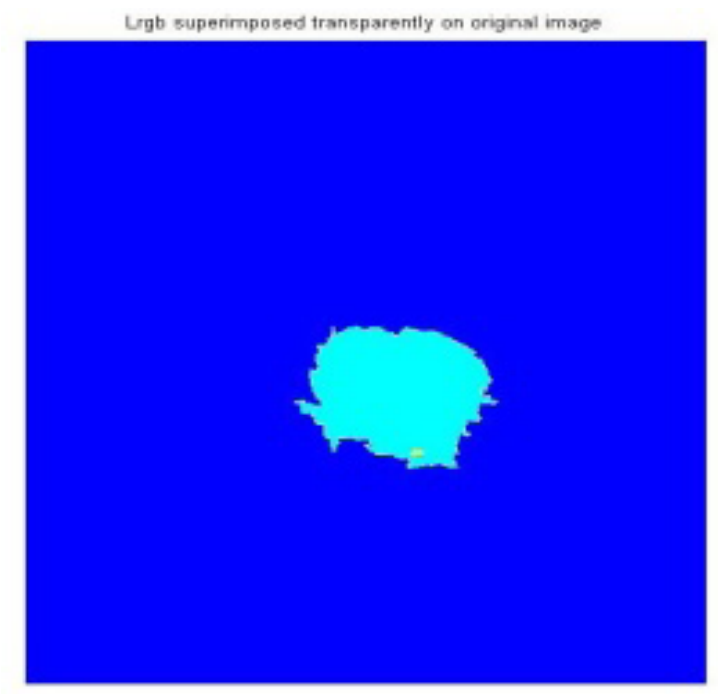

Figure 1. Superimposed transparently on original image.
The catchment bowls owning insignificant charge aren't stamped legitimately within the sight of commotion. Therefore, before applying the watershed set of tenets, pre-preparing step is utilized to take away the clamor and another sort of non-consistency from the test photograph while in transit to stamp best the favored catchment bowls. These stamped catchment bowls deliver the altered inclination photograph. Watershed revise is then connected to changed angle photograph to yield the last watershed edge follows.

The resultant picture is superimposed on the one of a kind result to show in Figure1, by the watershed algorithm, the broad partition comes with reference to the original image. The mammogram input image which is affected by larger calcium content is shown in Figure 2.

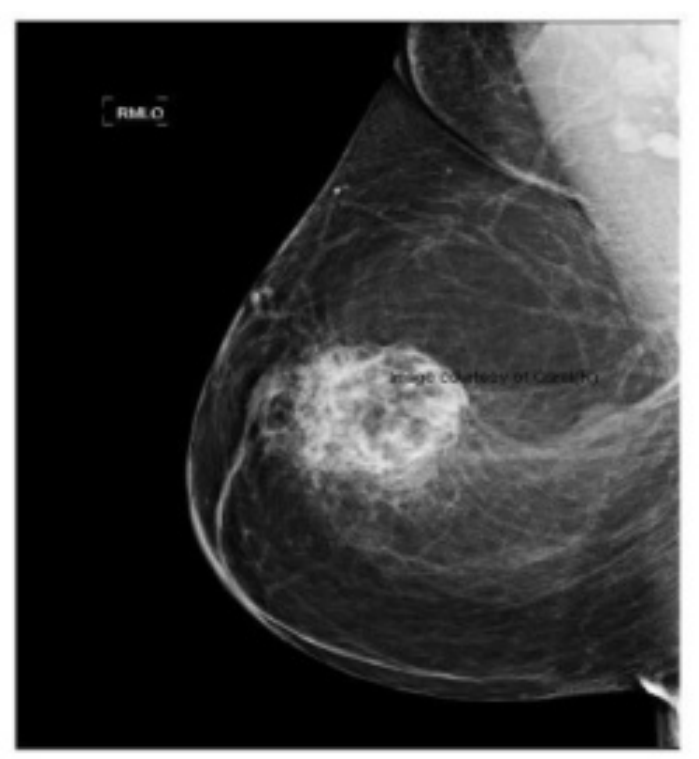

Figure 2. Input image.

\section{Result and Discussion}

The canny edge detector is the one which more convenient than the previous algorithm we discussed ${ }^{15}$. In canny edge detector the edges can be identified by the Laplace gradient operators. Three operators are there in the Laplace for estimating edges of the region. They are: 1 . Sobel Operator, 2. Robert Cross Gradient, and 3. Prewitt Operator. Among all three operators the sobel operator taken consideration to identify the calcium affected cell in the breast cancer image. The sobel operator mask is shown in Table 1. 
Table 1. Sobel Operators

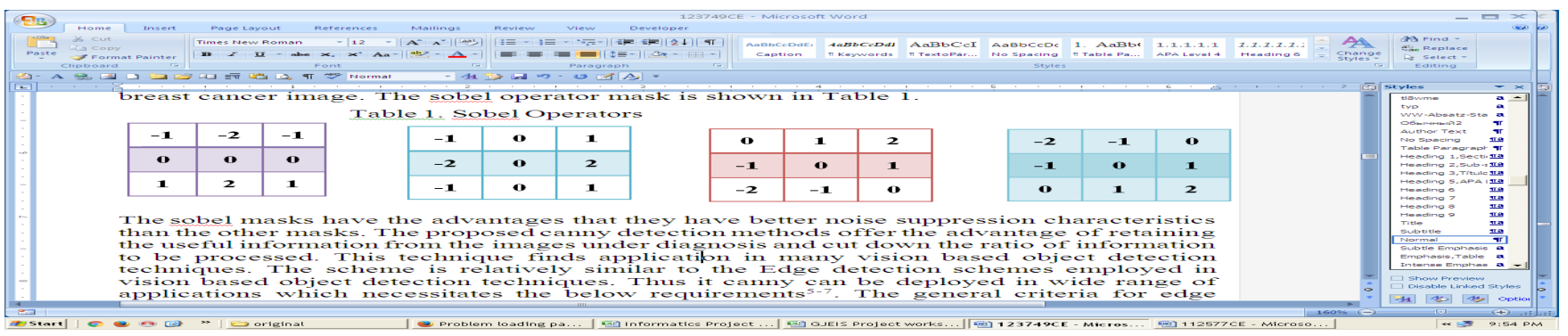

The sobel masks have the advantages that they have better noise suppression characteristics than the other masks. The proposed canny detection methods offer the advantage of retaining the useful information from the images under diagnosis and cut down the ratio of information to be processed. This technique finds application in many vision based object detection techniques. The scheme is relatively similar to the Edge detection schemes employed in vision based object detection techniques. Thus it canny can be deployed in wide range of applications which necessitates the below requirements ${ }^{5-7}$. The general criteria for edge location include:

1. Identification of edges which has the values lower than threshold, which suggests that the area should absolutely get however numerous edges showed up in the photo as could sensibly be normal,

\section{Original Image}

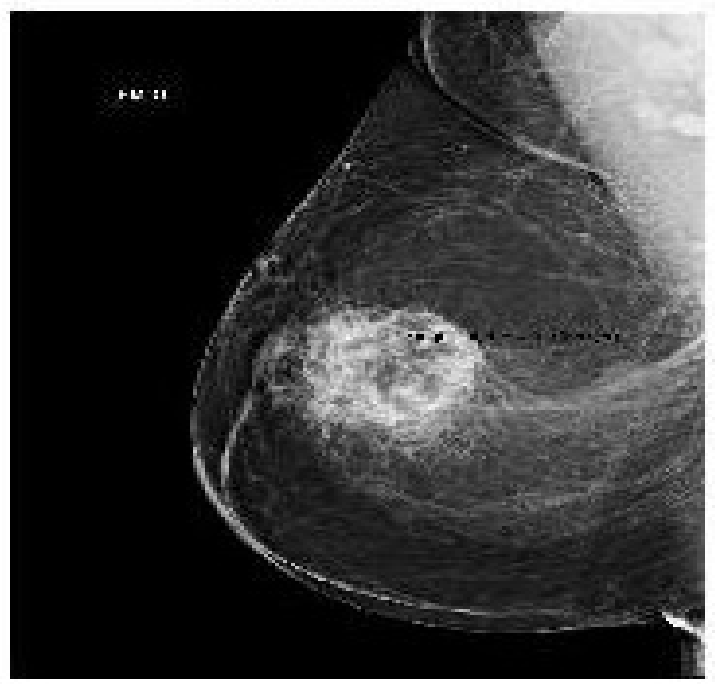

a. Original Image
2. The detected point of edge should be located within the central area of the edge, and

3. A given edge in the photo should simply be checked once, and where conceivable, picture clamor ought not to make false edges.

To satisfy these necessities Canny used the investigation of assortments - a technique which finds the limit which enhances a given utilitarian. The perfect limit in careful discoverer is delineated by the total of four exponential terms; be that as it may it can be approximated by the key backup of a Gaussian.

Among the edge area systems grew up to this point, canny edge disclosure computation is a champion among the most altogether described procedures that give awesome and tried and true recognizable proof. Inferable from its optimality to meet with the three criteria for

\section{Filtered Image}
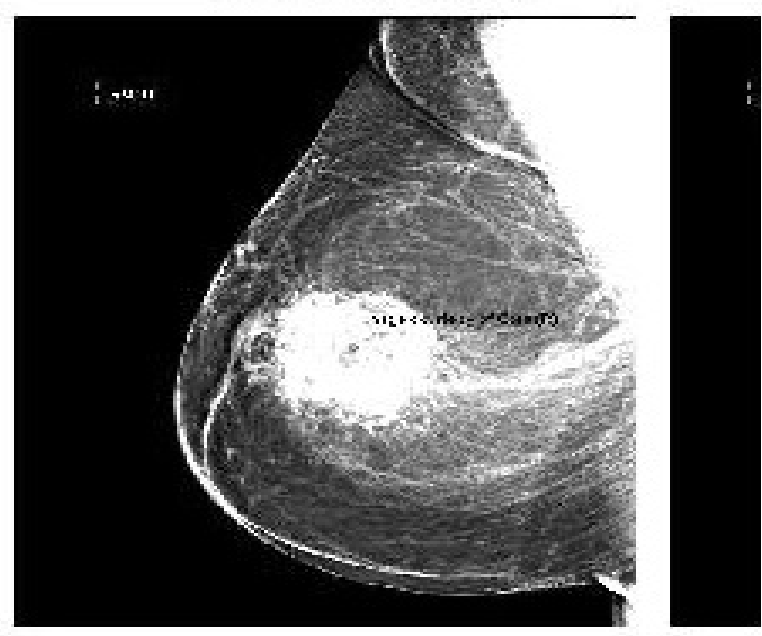

b. Filtered Image

Figure 3. Input and filtered image. 


\section{X-Direction Image}

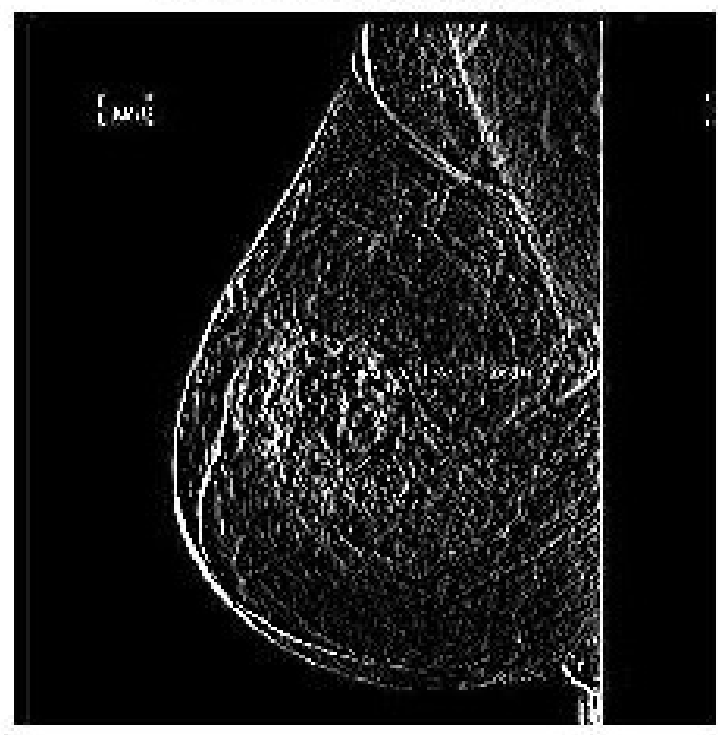

Figure 4. Sobel operator in $\mathrm{X}$ and $\mathrm{Y}$ direction.

edge distinguishing proof and the straightforwardness of process for use, it wound up a champion among the most acclaimed counts for edge disclosure. The Process of Canny edge location calculation can be 4 unique advances:

1. The filter is applied to the image in order to achieve the smooth pixel outputs. The filter preferred is Gaussian filter shown in Figure 3.

\section{Non-Max Suppress}

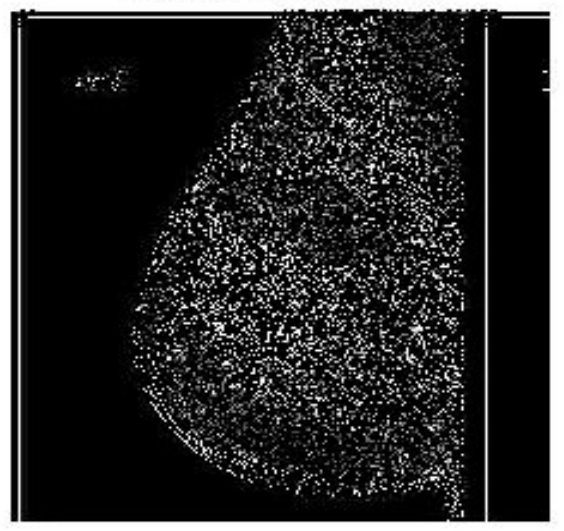

Figure 5. Non maximal suppression by canny.

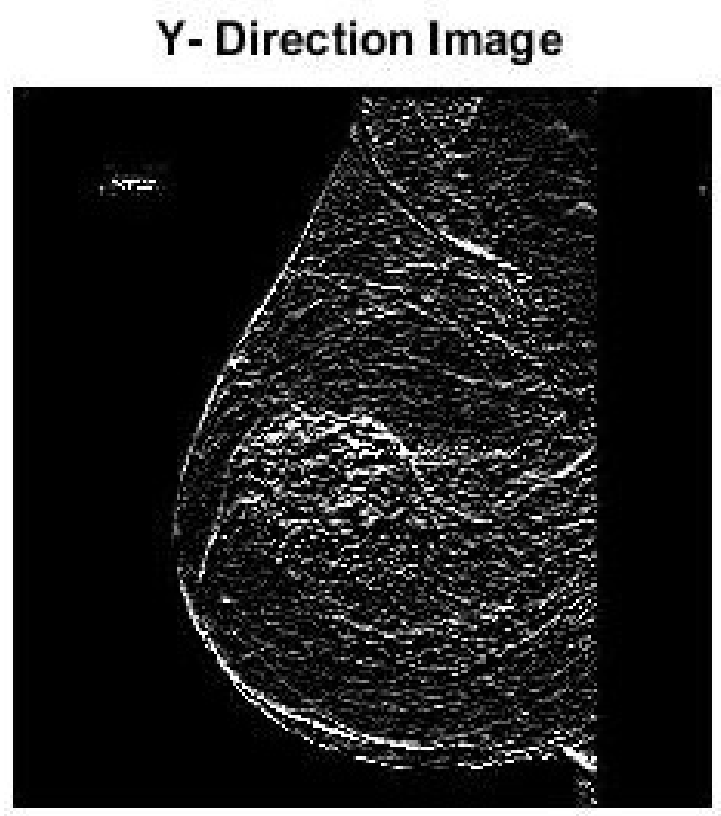

2. Among the pixels under investigation discover the indifferent gradient values. Thus the misleading identification of edges can be avoided, shown in Figure 4 and 5.

3. Two threshold values are preferred for the smooth edge identification.

4. Track edge by hysteresis: Finalize the detection of edges by suppressing all the other edges that are weak and not connected to strong edges, shown in Figure 6.

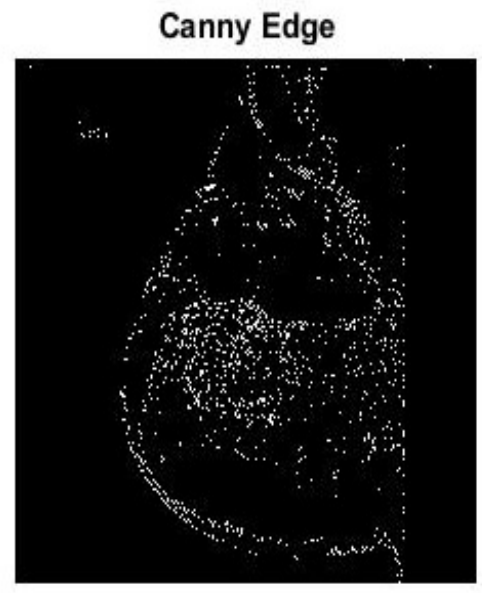

Figure 6. Canny detected calcium affected cells. 


\section{Conclusion}

In existing systems complex mathematical calculations are used and it is very difficult for the users to understand. Such complexity has been in need to provide accurate results in the specified area and also there may be chances for many false images i.e. samples not affected with cancer. We proposed canny edge detector to estimate the amount of affected cells. These algorithms are used to find the segmented regions accurately for different stages of patients. Watershed algorithm is better and simple way to identify the cancer cells.

\section{Reference}

1. Manikandan M, Andrews NV, Kavitha V. Investigation on Micro Calcifications of Brest Cancer from Mammogram Image Sequence, International Journal of Pure and Applied Mathematics. 2018; 118(20):645-49.

2. Balleyguier V, Vanel D, Athanasiou A, Mathieu MC, Sigal R. Breast Radiological Cases: Training with BI-RADS Classification, European Journal of Radiology. 2005; 54:97-106. Crossref. PMid: 15797298.

3. Radi MJ. Calcium oxalate crystals in breast biopsies, Archives of Pathology and Laboratory Medicine. 1989; 113(12):1367-69. PMid: 2589947.

4. Dengler J, Behrens S, Desaga J. Segmentation of micro calcification in mammograms, IEEE Transactions on Medical Imaging. 1993; 12:231-38. Crossref. PMid:18218457.

5. Przelaskowski A, Surowski P. Methods of medical image data optimization applied to archiving and telemedical transmission, Research Project of the State Commit-tee for Scientific Research No. 2002.
6. Betal D, Roberts N, Whitehouse G. Segmentation and numerical analysis of micro calcifications on mammograms using mathematical morphology, The British Journal of Radiology. 1997; 70:903-17. Crossref. PMid: 9486066.

7. Kim J, Park H. Statistical textural features for detection of micro calcifications in digitized mammograms, IEEE Transaction Medical Image. 1999; 18(3):231-38. Crossref. PMid:10363701.

8. Chany H, Sahiner B, Petrick N, Helvie M, Lam K, Adler D, Goodsitt M. Computerized classification of malignant and benign micro calcifications on mammograms: texture analysis using an artificial neural network, Physics in Medicine and Biology. 1997; 42:549-67. Crossref.

9. Kohonen T. Self-organizing maps in information sciences. Sprinter Series in Information Sciences. 1995, pp. 1-30. Crossref.

10. Dengler J, Behrens S, Desaga JF. Segmentation of micro calcifications in mammograms, IEEE Transactions on Medical Imaging. 1993; 12:634-42. Crossref. PMid:18218457.

11. Quadrades S, Sacristan A. Automated extraction of micro calcifications BI-RADS numbers in mammograms, International Conference on Image Processing. 2001; 2:289-92.

12. Shen L, Rangayyan R, Desautels J. Shape analysis of mammographic calcifications. Proceedings 5th Annual IEEE Symposium on Computer-Based Medical Systems; 1992. p. 123-28. Crossref.

13. Jiang Y. Classification of breast lesions from mammograms. Handbook of Medical Imaging; 2000. p. 341-57. Crossref.

14. Manikandan M, Prabakar S, Porkumaran K. Active Cardiac Model and its structure revealing from Ultrasound Images, International Journal of Engineering Research and General Science. 2014; 2(4). 